Urologe 2014 · 53:1065

DOI 10.1007/s00120-014-3569-2

Online publiziert: 5. Juli 2014

(c) Springer-Verlag Berlin Heidelberg 2014

\title{
H. Rübben
}

Klinik und Poliklinik für Urologie, Uroonkologie und Kinderurologie, Universitätsklinikum Essen

\section{Nahrungsergänzung mit Sojaproteinisolat}

Gabe von Sojaprotein Isolat gegen ein Placebo untersucht. 87 Männer erhielten $20 \mathrm{~g}$ des Proteins, 90 ein Placebo. Nach 2 Jahren fand sich ein biochemisches Rezidiv bei $27,2 \%$ der Männer in der Behandlungsgruppe und 29,5\% der Placebogruppe. Die Autoren kommen zu dem Schluss, dass Sojaprotein Isolat das Risiko nach radikaler Prostatektomie ein Rezidiv zu entwickeln, nicht beeinflusst.

\section{Korrespondenzadresse}

Prof. Dr. Dr. h.c H. Rübben

Urologische Universitätsklinik Essen,

Hufelandstraße 55, 45122 Essen

herbert.ruebben@uni-duisburg-essen.de

Interessenkonflikt. H. Rübben gibt an, dass kein Interessenkonflikt besteht.
In einer randomisierten Untersuchung bei Männern nach radikaler Prostatektomie mit einem hohen Risiko für ein Rezidiv wurde doppelblind bei 177 Männern die
Urologe 2014 · 53:1065

DOI 10.1007/s00120-014-3571-8

Online publiziert: 5. Juli 2014

c) Springer-Verlag Berlin Heidelberg 2014

\section{H. Rübben}

Klinik und Poliklinik für Urologie, Uroonkologie und Kinderurologie, Universitätsklinikum Essen

\section{Adipositas und tödliches Prostatakarzinom}

kung oder waren an ihrem Tumor verstorben. Das Risiko, eine solche Erkrankung zu entwickeln, war statistisch höher in der Gruppe der adipösen Patienten, insbesondere dann, wenn in dem Tumor die Genfusion TMPRSS2:ERG nachgewiesen werden konnte.

TMPRSS2:ERG wurde 2005 entdeckt und ist deshalb bedeutsam, weil sie ein Modell hormoneller Regulation eines Onkogens darstellt. Bedeutsam ist in diesem Zusammenhang die Beobachtung, dass die Genfusion dann eine schlechtere Prognose signalisiert, wenn die Männer mit einer nicht kurativen Intention behandelt wurden nicht aber, wenn sie radikal prostatektomiert wurden [1]. Diese Ergebnisse wurden in einem systematischen Review von prospektiven Kohortenstudien ebenfalls belegt $[2,3]$.

\section{Literatur}

1. Demichelis $F$ et al (2007) TMPRSS2:ERG gene fusion associated with lethal prostate cancer in a watchful waiting cohort. Oncogene 26:4596-9

2. Golabeck T et al (2014) Obesity and Prostate Cancer Incidence and Mortality: A Systematic Review of Prospective Cohort Studies. Urol Int 92:7-14

3. Pettersson A et al (2013) Modification of the Association Between Obesity and Lethal Prostate Cancer by TMPRSS2:ERG. JNCI 105:1881-1890

\section{Korrespondenzadresse}

Prof. Dr. Dr. H. Rübben

Klinik und Poliklinik für Urologie, Uroonkologie und Kinderurologie, Universitätsklinikum Essen, Hufelandstraße 55, 45122 Essen

herbert.ruebben@uni-duisburg-essen.de

Interessenkonflikt. H. Rübben gibt an, dass kein Interessenkonflikt besteht. 